\title{
Densidade Mineral Óssea de Crianças e Adolescentes com Hipotireoidismo Congênito
}

\section{artigo original}

\author{
ADRIANE DE A.C. DEMARTINI \\ CAROLINA A.M. KULAK \\ VICTORIA C. BORBA \\ MÔNICA N.L. CAT \\ ROBERTA S. DONDONI \\ ROMOLO SANDRINI \\ SUZANA NESI-FRANÇA \\ LUIZ DE LACERDA FILHO
}

Unidade de Endocrinologia

Pediátrica (UEP) e Serviço de

Endocrinologia e Metabologia

do Hospital de Clínicas da

Universidade Federal do Paraná

(UFPR), Curitiba, PR.
Recebido em 04/12/06

Revisado em 26/03/07 Aceito em 28/05/07

\section{RESUMO}

Realizou-se estudo transversal com 60 pacientes $(9,9 \pm 1,8$ anos) com hipotireoidismo congênito ( $\mathrm{HC}$ ) (grupo $\mathrm{A}$ ): 40 meninas (23 pré-púberes) e 20 meninos (18 pré-púberes), com grupo controle (grupo B) constituído por 28 indivíduos $(10,4 \pm 2,1$ anos): 18 meninas (8 pré-púberes) e 10 meninos (9 pré-púberes). Objetivos: Avaliar a densidade (DMO) e o conteúdo mineral ósseo (CMO) e correlacioná-los com idade cronológica e óssea (IO), sexo, maturação sexual, dose de $l-\mathrm{T}_{4}, \mathrm{TSH}, \mathrm{TT}_{4}, \mathrm{FT}_{4}$, e etiologia do HC. IO, DMO e CMO de corpo total (DXA) foram obtidos dos 2 grupos; $\mathrm{TSH}_{1} \mathrm{TT}_{4}$ e $\mathrm{FT}_{4}$, apenas dos pacientes. $\mathrm{DMO}$ foi menor no grupo $\mathrm{A}$ $\left(0,795 \pm 0,075 \mathrm{~g} / \mathrm{cm}^{2}\right.$ vs. $\left.0,832 \pm 0,092 ; \mathrm{p}=0,04\right)$ e maior nas meninas púberes do que nas pré-púberes $(p=0,004)$. Não houve diferença significativa de DMO e CMO quanto ao sexo e etiologia do HC. Nosso estudo mostra que a DMO foi significativamente menor no grupo com $\mathrm{HC}$, diferente dos dados da literatura. (Arq Bras Endocrinol Metab 2007;51/7:1084-1092)

Descritores: Hipotireoidismo congênito; Densidade mineral óssea; Conteúdo mineral ósseo; Absorciometria por dupla emissão de raios $X$ (DXA)

\section{ABSTRACT}

Bone Mineral Density of Children and Adolescents with Congenital Hypothyroidism.

A cross sectional study was made on 60 patients $(9.9 \pm 1.8 \mathrm{yr}$-old) with congenital hypothyroidism $(\mathrm{CH})$ (group A): 40 girls (23 prepubertal) and 20 boys (18 prepubertal). Control group (group B) was constituted of 28 healthy children $(10.4 \pm 2.1 \mathrm{yr}$-old): 18 girls ( 8 prepubertal) and 10 boys (9 prepubertal). Aims: To evaluate bone mineral density (BMD) and content (BMC) and to correlate them with chronological and bone age (BA), sex, sexual maturation, $I-\mathrm{T}_{4}$ dose, $\mathrm{TSH}, \mathrm{TT}_{4}, \mathrm{FT}_{4}$, and $\mathrm{CH}$ etiology. $\mathrm{BA}$, total body $B M D$, and BMC (DXA) were obtained of both groups. $\mathrm{TSH}_{1} \mathrm{TT}_{4}$, and $\mathrm{FT}_{4}$ were measured in patients only. BMD was lower in group $A(0.795 \pm 0.075$ $\mathrm{g} / \mathrm{cm}^{2}$ vs. $\left.0.832 \pm 0.092 ; \mathrm{p}=0.04\right)$ and higher in pubertal than in prepubertal girls $(p=0.004)$. There was no significant difference between BMD and BMC related to sex and $\mathrm{CH}$ etiology. Our data demonstrated that BMD was significantly lower in children with $\mathrm{CH}$, different from what has been published in the literature. (Arq Bras Endocrinol Metab 2007;51/7:1084-1092)

Keywords: Congenital hypothyroidism; Bone mineral density; Bone mineral content; Dual-energy X-ray absorptiometry (DXA) 
$\mathrm{O}$ HIPOTIREOIDISMO CONGÊNITO (HC) é um dos distúrbios endócrinos mais freqüentes, ocorrendo em aproximadamente $1: 3000$ a $1: 4000$ nascimentos e é uma das causas preveníveis mais comuns de retardo mental $(1,2)$.

A implantação de Programas de Triagem Neonatal, a partir da década de 70, mudou a evolução da doença, e crianças com HC são atualmente identificadas e tratadas precocemente com levotiroxina $\left(l-\mathrm{T}_{4}\right)$, o que pode ter efeitos na formação e no metabolismo ósseo.

Diferentes mecanismos de ação dos hormônios tireoideanos (HT) sobre o tecido ósseo já foram propostos e foram recentemente revisados por Harvey e cols. (3) e Basset e Williams (4). A triiodotironina $\left(\mathrm{T}_{3}\right)$ influencia a produção de citocinas, fatores de crescimento e marcadores da remodelação óssea, e seus receptores nucleares (TR $\alpha$ e TR $\beta$ ) podem ser encontrados em osteoblastos, células estromais da medula óssea e condrócitos da placa de crescimento $(5,6)$. Na cartilagem de crescimento, a $\mathrm{T}_{3}$ inibe a proliferação de condrócitos, mas estimula sua diferenciação. Estimula a proliferação, a diferenciação e a apoptose osteoblástica e aumenta a síntese de interleucina-6 (IL-6), de prostaglandinas e do ligante do receptor ativador do fator nuclear kappa B (RANKL) pelos osteoblastos, o que estimula a osteoclastogênese. Acrescenta-se que a IL-6 foi encontrada em concentrações altas em pacientes com hipertireoidismo (7).

Estudos demonstraram deficiência de calcitonina (CT), tanto basal quanto após estímulo (cálcio, pentagastrina), em pacientes com $\mathrm{HC}$, em relação ao grupo controle, sugerindo que fatores tróficos secretados pelas células foliculares são necessários para o desenvolvimento adequado das células C (8-11). Entretanto, até o momento, poucos autores estudaram a massa óssea em crianças e adolescentes com HC, e os estudos existentes mostram DMO normal e semelhante à dos controles na coluna lombar (12-16), fêmur $(12,14,15)$ e falanges proximais (16). Estes autores concluíram que o tratamento prolongado e cuidadosamente monitorizado com altas doses de $l-\mathrm{T}_{4}$ não causariam a diminuição da massa óssea na infância e, se houvesse um efeito deletério sobre o esqueleto, o crescimento longitudinal teria reparado o dano com sucesso até a idade dos pacientes destes estudos (1216). Por sua vez, Demeester-Mirkine e cols. (10) encontraram conteúdo e densidade mineral na diáfise do rádio mais baixos nas sete mulheres com $\mathrm{HC}$ quando comparadas aos controles de mesmo sexo e idade (10\% de redução no CMO e 7\% na DMO). Como os mesmos autores (11) encontraram baixos níveis de CT (basal e estimulada), quando comparados ao grupo controle, sugeriu-se que a deficiência de CT precoce no HC teria prejudicado o desenvolvimento esquelético e teria contribuído para a diminuição da massa óssea. No entanto, Demeester-Mirkine e cols. (10) não dispunham dos valores de $\mathrm{T}_{4} \mathrm{e}$ TSH durante todo o período de tratamento, não sendo possível determinar se a perda de massa óssea teria sido induzida pelo excesso de HT.

Como há poucos estudos, e todos com pequeno número de pacientes, e nenhum utilizando a densitometria óssea de corpo total, considerado método mais adequado até o momento para a avaliação da massa óssea nesta faixa etária, realizou-se o presente estudo com os objetivos de avaliar a DMO e o conteúdo mineral ósseo (CMO) em crianças e adolescentes com $\mathrm{HC}$ diagnosticado por triagem neonatal e correlacionar com idade cronológica (IC), idade óssea (IO), sexo, maturação sexual, índice de massa corporal (IMC), renda familiar per capita, escolaridade dos pais, ingestão diária de cálcio, prática de atividade física, dose de $l-\mathrm{T}_{4}$, níveis de TSH, de $\mathrm{T}_{4}$ total $\left(\mathrm{TT}_{4}\right)$ e $\mathrm{T}_{4}$ livre $\left(\mathrm{FT}_{4}\right)$ e etiologia do $\mathrm{HC}$. Os resultados obtidos foram comparados aos de um grupo controle.

\section{MATERIAL E MÉTODOS}

Foi realizado um estudo transversal com pacientes em acompanhamento na Unidade de Endocrinologia Pediátrica (UEP) do Hospital de Clínicas da UFPR a partir de 1991. Densitometria óssea, exames laboratoriais e último exame físico foram realizados no período de fevereiro a outubro de 2005.

O estudo foi aprovado pelo Comitê de Ética em Pesquisa em Seres Humanos do Hospital de Clínicas da UFPR. Termo de consentimento livre e esclarecido foi explicado e fornecido no dia de realização da densitometria óssea, sendo assinado pela criança ou adolescente e pelo seu responsável maior de idade.

\section{População de estudo (grupo A)}

Constitui-se de 60 pacientes com HC primário permanente diagnosticados pelo Teste de Triagem Neonatal realizado pela Fundação Ecumênica de Proteção ao Excepcional no Estado do Paraná e confirmado por níveis baixos de $\mathrm{T}_{4} \mathrm{e}$ altos de TSH antes do início do tratamento e no momento de realização da cintilografia de tireóide para investigação etiológica. Foram selecionados conforme compareciam para a consulta previamente agendada, respeitando os critérios de exclusão do estudo.

Dados dos prontuários foram coletados desde a primeira consulta de cada paciente até a data de realização da densitometria óssea e último exame clínico, sendo analisadas as seguintes variáveis: $\mathrm{TT}_{4}$ e TSH pré-tratamento, idade de início do tratamento, valores de $\mathrm{TSH}, \mathrm{TT}_{4}$ e $\mathrm{FT}_{4}$ durante o 
tratamento, dose de $l-\mathrm{T}_{4}(\mu \mathrm{g} / \mathrm{kg} / \mathrm{dia})$ inicial e a cada consulta, cintilografia de tireóide, estatura e escolaridade dos pais e renda familiar per capita.

Para investigação etiológica, a partir dos trinta meses de idade, todos os pacientes tiveram a medicação suspensa durante, no mínimo, trinta dias. Após este período, realizouse exame clínico, coleta de sangue para dosagem sérica de $\mathrm{TT}_{4}$ e/ou $\mathrm{FT}_{4}$ e TSH e cintilografia de tireóide ( ${ }^{131} \mathrm{I}$ ou ${ }^{123} \mathrm{I}$ ).

Em praticamente todas as consultas, dosagens séricas de TSH, $\mathrm{TT}_{4} \mathrm{e} / \mathrm{ou} \mathrm{FT}_{4}$ foram realizadas. Para análise estatística, a fim de se evitar a individualização dos resultados, valores de TSH, obtidos durante o seguimento, maiores ou iguais a $50 \mu \mathrm{U} / \mathrm{ml}$ foram excluídos (29 valores, de pacientes distintos e consultas em idades diferentes), quando esses resultados não estavam compatíveis com valores de $\mathrm{TT}_{4}$ $\mathrm{e} /$ ou $\mathrm{FT}_{4}$ ou resultavam da interrupção não recomendada da $l-\mathrm{T}_{4}$, ou seja, TSH alto, com níveis normais de $\mathrm{TT}_{4} \mathrm{e} / \mathrm{ou}$ $\mathrm{FT}_{4}$. Foram calculadas as médias de $\mathrm{TT}_{4} \mathrm{e}$ TSH durante todo o período de acompanhamento, excluindo os valores das ocasiões acima citadas. Apesar de a dosagem do TSH ser útil como triagem para identificar pacientes com disfunção tireoideana, um paciente não deve iniciar tratamento com base somente no nível anormal de TSH, pois este pode aumentar em pacientes agudamente enfermos e altera-se mais rapidamente se o uso de $l$ - $\mathrm{T}_{4}$ for irregular.

Baseando-se nestas informações, a média de $\mathrm{TT}_{4}$ foi utilizada para classificar o controle laboratorial como:

a) adequado: média de $\mathrm{TT}_{4}$ maior ou igual a $8,0 \mu \mathrm{g} / \mathrm{dl}$;

b) inadequado: média de $\mathrm{TT}_{4}$ menor que $8,0 \mu \mathrm{g} / \mathrm{dl}$.

\section{Critérios de inclusão}

a) idade entre 7 e 14 anos;

b) diagnóstico de HC primário permanente;

c) início do tratamento com $l-\mathrm{T}_{4}$ até o $120^{\circ}$ dia de vida.

\section{Critérios de exclusão}

a) início do tratamento após o $120^{\circ}$ dia de vida;

b) déficit cognitivo que prejudicasse a colaboração do paciente para a realização da densitometria óssea;

c) uso de drogas anticonvulsivantes ou diuréticos, glicocorticóides, suplementos vitamínicos ou de cálcio, somatropina recombinante humana ou de análogo do hormônio liberador de gonadotrofinas;

d) doença grave concomitante;

e) restrição prolongada no leito (mais de 3 meses);

f) suspensão da $l$-T 4 por período igual ou maior que 6 meses.

\section{Avaliação clínica}

Exame clínico foi realizado no dia em que foi feita a densitometria óssea. Peso $(\mathrm{P})$ e estatura $(\mathrm{E})$ foram verificados em todos os pacientes, utilizando uma balança Filizola ${ }^{\circledR}$ (São Paulo, SP) e um estadiômetro fixo em parede (Stadiometer Mode S100, Ayrton Corporation ${ }^{\circledR}$, Prior Lake, Minesota), respectivamente. O índice de massa corporal (IMC) foi calculado pela fórmula de Quetelet $\left(\mathrm{IMC}=\mathrm{P} \div \mathrm{E}^{2}\right)$. Escore- $\mathrm{Z}$ do peso, da estatura e do IMC foram utilizados para cálculos estatísticos e foram calculados através do Programa NutStat do
Epi Info $^{\mathrm{TM}}$ versão 3.3 .2 (Fevereiro 2005), utilizando como referência os dados do NCHS (17). O escore-Z da estatura dos pais também foi calculado através do mesmo programa.

Estadiamento puberal (18) e mensuração do volume testicular nos meninos com orquidômetro de Prader (19) foram realizados sempre pelo mesmo examinador.

Também aplicou-se um questionário que inclui cor da pele, uso de vitamina $\mathrm{D}$ no primeiro ano de vida, atividade física, ingestão diária de cálcio, antecedentes de fraturas e antecedentes mórbidos, baseando-se no modelo desenvolvido por Thériault (20).

Devido à grande miscigenação racial existente no Brasil, para cor da pele utilizou-se os termos branca e não branca, sendo que esta incluiu indivíduos negros, pardos e mulatos.

A ingestão média diária de cálcio foi baseada nas informações dos pacientes e de seus responsáveis com relação ao consumo de leite e derivados. Utilizou-se a classificação norteamericana, considerando a ingestão como adequada quando maior que $800 \mathrm{mg} /$ dia para crianças de 7 a 9 anos incompletos, e maior que $1300 \mathrm{mg} /$ dia para crianças e adolescentes de 9 a 14 anos e, como inadequada, se abaixo destes níveis (21).

A prática de atividade física foi considerada como regular quando realizada em tempo igual ou superior a três horas por semana.

\section{Exames de análises clínicas}

Dosagem sérica de cálcio iônico $\left(\mathrm{Ca}^{+2}\right)$, creatinina, fósforo inorgânico (Pi), fosfatase alcalina total (FA), $\mathrm{TT}_{4}, \mathrm{FT}_{4}$ e TSH foi realizada no Laboratório de Análises Clínicas do Hospital de Clínicas da UFPR, somente nos pacientes com HC (grupo A) no dia de realização da densitometria. A tabela 1 mostra os valores de referência e o método utilizado para cada exame.

\section{Exames de imagem e densitometria óssea}

Radiografia de mãos e punhos para avaliação da idade óssea (IO) foi realizada no Serviço de Radiologia do Hospital de Clínicas da UFPR e analisada conforme os critérios de Greulich e Pyle (22) por um mesmo observador.

O método utilizado para a mensuração do CMO e da DMO foi a absorciometria por dupla emissão de raios $\mathrm{X}$ (DXA). Esta foi mensurada com a criança ou o adolescente em posição supina, utilizando-se o aparelho HOLOGIC QDR 1000 W (Hologic Inc. ${ }^{\circledR}$, Waltham, Massachusetts, USA) do Serviço de Endocrinologia e Metabologia do Paraná (SEMPR) do Hospital de Clínicas da UFPR, por técnico especializado, e os resultados foram analisados por um único observador. Os valores absolutos da área corporal total, em $\mathrm{cm}^{2}$, do CMO, em gramas (g), e da DMO, em $\mathrm{g} / \mathrm{cm}^{2}$, foram obtidos e analisados.

\section{Grupo-controle (grupo B)}

Os resultados obtidos na avaliação clínica e densitométrica dos pacientes com HC foram comparados com os resultados obtidos a partir de um grupo-controle, denominado grupo B.

Foram selecionados 28 indivíduos (18 meninas) saudáveis, de mesmo grupo etário e de mesma classe sócio- 
Tabela 1. Métodos e valores de referência dos exames laboratoriais.

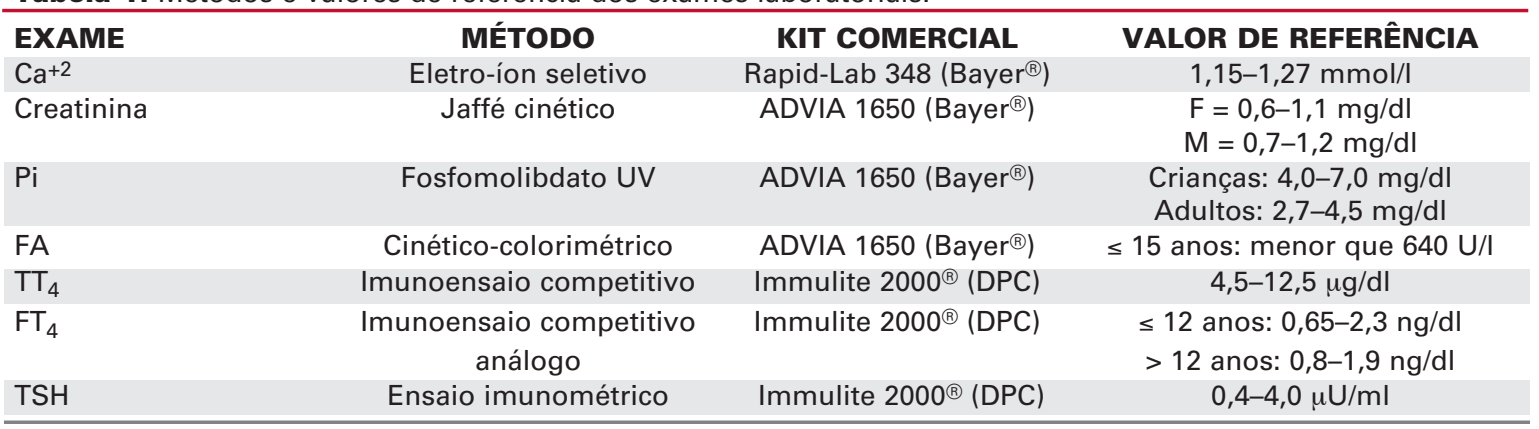

econômica, sem evidência de doença óssea, renal ou tireoideana e sem uso de drogas que pudessem interferir no metabolismo ósseo ou de suplementos vitamínicos há pelo menos um ano antes da convocação. Foram incluídos somente indivíduos que realizaram Teste de Triagem Neonatal para HC. Pacientes e controles foram pareados com relação ao sexo e estadiamento puberal.

Todos os pais, crianças e adolescentes assinaram o termo de consentimento, e os indivíduos deste grupo realizaram, além da anamnese, avaliação clínica, radiografia de mãos e punhos para avaliação da IO e avaliação densitométrica pela DXA.

\section{Análise estatística}

O tamanho da amostra foi estimado considerando um erro de tipo I de $5 \%$ (alfa) e erro do tipo II de $10 \%$, com um poder de teste estimado mínimo de 90\%.

A diferença entre as variáveis contínuas foi avaliada através dos testes $t$ de Student para amostra independente ou dependente e ANOVA de acordo com a natureza dos grupos estudados. Para avaliar o grau de associação entre variáveis contínuas de distribuição normal foi utilizada a análise de correlação de Pearson. A diferença entre freqüências foi avaliada através do teste qui-quadrado de Pearson. Para todos foram utilizados os testes bicaudais, considerando que as diferenças poderiam estar distribuídas para ambos os lados da curva, com nível de significância mínimo de $5 \%$.

\section{RESULTADOS}

Sessenta pacientes foram incluídos no grupo A, sendo $40(66,7 \%)$ do sexo feminino e $20(33,3 \%)$ do sexo masculino. A média da IC foi de $9,9 \pm 1,8$ anos, enquanto a da IO foi de 9,6 $\pm 2,2$ anos $(\mathrm{p}=0,16)$. Trinta e duas crianças $(53,3 \%)$ tinham cor da pele branca e $28(46,7 \%)$, não branca. A maioria $(86,7 \%)$ dos pacientes tinha renda familiar inferior a 2 saláriosmínimos per capita. Houve predomínio da escolaridade em nível fundamental tanto para as mães $(66,7 \%)$ quanto para os pais $(70 \%)$.
$\mathrm{O}$ uso de vitamina $\mathrm{D}$ no primeiro ano de vida foi relatado em 24 crianças $(40 \%)$ e a história de fratura, em quatro crianças $(6,7 \%)$, sendo todas traumáticas, de membros superiores e com cura sem seqüelas.

A mediana da ingestão diária de cálcio foi de $700 \mathrm{mg}$, variando de 150 a $2156 \mathrm{mg}$. Trinta e nove crianças $(65 \%)$ apresentaram ingestão diária de cálcio inadequada e 21 (35\%), adequada, conforme as recomendações norte-americanas (21). Apenas 14 pacientes $(23,3 \%)$ relataram atividade física regular.

A idade de início do tratamento foi, em média, $32,8 \pm 19,9$ dias de vida. Antes do início do tratamento, a mediana do TSH $(\mu \mathrm{U} / \mathrm{ml})$ era de 106 , variando de 19,1 a 867 e a mediana do $\mathrm{TT}_{4}(\mu \mathrm{g} / \mathrm{dl})$ era de 2,26, variando de 0,2 a 11,2 . Os valores medianos de $\mathrm{TSH}(\mu \mathrm{U} / \mathrm{ml})$ e $\mathrm{TT}_{4}(\mu \mathrm{g} / \mathrm{dl})$ no momento de realização da cintilografia de tireóide foram, respectivamente, 162, variando de 12,2 a 726 e 1,7 , variando de 0,3 a 11,7 .

Vinte e seis pacientes $(43,3 \%)$ apresentavam ectopia de tireóide; 21 (35\%), agenesia; 4 (6,7\%), hipoplasia e 9 (15\%), defeitos na síntese hormonal (disormonogênese).

Durante todo o seguimento, a dose média de $l$ $\mathrm{T}_{4}$ foi $5,5 \pm 0,8 \mu \mathrm{g} / \mathrm{kg} /$ dia; a mediana do $\mathrm{TSH}$ $(\mu \mathrm{U} / \mathrm{ml})$ foi de 5,4 , variando de 1,1 a 12,4 ; a média do $\mathrm{TT}_{4}(\mu \mathrm{g} / \mathrm{dl})$ foi $13,1 \pm 1,2$ e a do $\mathrm{FT}_{4}(\mathrm{ng} / \mathrm{dl})$, $1,66 \pm 0,16$. Com base na média de $\mathrm{TT}_{4}$ durante todo o seguimento, todas as crianças apresentaram controle laboratorial adequado.

A dose média inicial de $l-\mathrm{T}_{4}$ foi de $11,7 \pm 1,8$ $\mu \mathrm{g} / \mathrm{kg} /$ dia e diminuiu gradativamente. No momento da densitometria óssea, a média foi de $3,1 \pm 0,9 \mu \mathrm{g} / \mathrm{kg} /$ dia.

\section{Avaliação clínica e laboratorial na ocasião da densitometria óssea}

As médias \pm DP da estatura, do peso e do IMC, com os respectivos escores- $\mathrm{Z}$, dos pacientes do grupo $\mathrm{A}$, na ocasião da densitometria óssea, estão apresentadas na tabela 
2. A análise estatística não mostrou diferença significativa dos parâmetros avaliados entre meninas e meninos. As medianas do escores- $Z$ da estatura das mães e dos pais foi $-0,78(-3,12-+1,34)$ e $-0,96(-2,75-+1,43)$, respectivamente. Ao aplicar-se o teste de Wilcoxon, observouse que as meninas tinham escore- $Z$ da estatura significativamente maior que os escores- $Z$ da estatura de suas mães $(\mathrm{p}=0,007)$ e de seus pais $(\mathrm{p}=0,001)$, assim como os meninos em relação às suas mães $(\mathrm{p}=0,01)$ e aos seus pais $(\mathrm{p}=0,001)$.

Vinte e três $(38,4 \%)$ meninas e $18(30 \%)$ meninos eram pré-púberes. A média de idade de início da puberdade foi de $10,0 \pm 1,2$ anos nas dezessete meninas, enquanto que dois meninos iniciaram a puberdade aos 10 e aos 10,6 anos. Cinco meninas (8,3\%) já apresentavam estádio mamário M5 e nenhum menino tinha a puberdade completa.

As médias \pm DP dos exames laboratoriais por ocasião da densitometria estão apresentadas na tabela 3. Todos os pacientes apresentavam creatinina sérica normal.

\section{Densitometria óssea}

Os valores (média $\pm \mathrm{DP})$ de área corporal total $\left(\mathrm{cm}^{2}\right)$, CMO $(\mathrm{g})$ e DMO $\left(\mathrm{g} / \mathrm{cm}^{2}\right)$ foram $1180,95 \pm 305,64$, $959,53 \pm 343,57$ e $0,795 \pm 0,075$, respectivamente.
Não houve correlação entre peso de nascimento e $\operatorname{DMO}(\mathrm{r}=0,31)$ ou CMO $(\mathrm{r}=0,34)$. Também não se encontrou correlação entre $\mathrm{DMO}$ e valores iniciais de TSH $(r=-0,13)$ e $\mathrm{TT}_{4}(\mathrm{r}=-0,24)$, assim como entre CMO e estas variáveis (TSH inicial: $r=-0,21$ e $\mathrm{TT}_{4}$ inicial: $\left.\mathrm{r}=-0,33\right)$.

A DMO e o CMO aumentaram com a idade, principalmente quando se considerou a IO. Houve uma correlação positiva da DMO com a IC $(r=0,60)$ e com a IO $(r=0,75)$, e do CMO com a IC $(r=0,72)$ e com a $\mathrm{IO}(\mathrm{r}=0,87)$.

Não houve diferença significativa de DMO entre os sexos, tanto ao se considerar todos os pacientes (meninas: $0,800 \pm 0,087$ vs. meninos: 0,786 $\pm 0,049$ $\left.\mathrm{g} / \mathrm{cm}^{2} ; \mathrm{p}=0,52\right)$, quanto ao se considerar somente as crianças pré-púberes $(0,766 \pm 0,060$ vs. $0,780 \pm 0,047$ $\left.\mathrm{g} / \mathrm{cm}^{2} ; \mathrm{p}=0,44\right)$. O mesmo ocorreu ao se analisar o CMO (todas as meninas: $995,8 \pm 387,0$ vs. todos os meninos: $887,1 \pm 225,4 \mathrm{~g} ; \mathrm{p}=0,25$; meninas prépúberes: $802,7 \pm 220,7$ vs. meninos pré-púberes $846,4 \pm 198,1$ g; p = 0,51). Não houve correlação significativa da DMO e do CMO com o grau de escolaridade materna (DMO: $\mathrm{p}=0,70 ; \mathrm{CMO}: \mathrm{p}=0,85$ ) ou paterna (DMO: $\mathrm{p}=0,29$; CMO: $\mathrm{p}=0,30)$ nem com a renda familiar per capita (DMO: $\mathrm{p}=0,40$; CMO: $\mathrm{p}=0,49)$.

Tabela 2. Medidas dos pacientes do grupo A.

\begin{tabular}{lccc}
\hline Parâmetro & $\begin{array}{c}\text { Sexo Feminino } \\
(\mathbf{n}=\mathbf{4 0})\end{array}$ & $\begin{array}{c}\text { Sexo Masculino } \\
(\mathbf{n}=\mathbf{2 0})\end{array}$ & $\begin{array}{c}\mathbf{p} \\
\text { Estatura }(\mathrm{cm})^{(1)}\end{array}$ \\
$137,1 \pm 13,3$ & $135,0 \pm 9,6$ & (3) 0,52 \\
Escore-Z da estatura(2) & $-0,30(-2,02-+2,79)$ & $-0,55(-1,93-+1,59)$ & (4) 0,32 \\
Peso $(\mathrm{kg})^{(1)}$ & $34,8 \pm 10,2$ & $31,8 \pm 6,5$ & (3) 0,23 \\
Escore-Z do peso(2) & $+0,28(-1,72-+2,34)$ & $+0,16(-1,87-+1,20)$ & (4) 0,40 \\
IMC $\left(\mathrm{kg} / \mathrm{m}^{2}\right)^{(1)}$ & $18,1 \pm 2,7$ & $17,2 \pm 1,9$ & (3) 0,20 \\
Escore-Z do IMC(2) & $+0,36(-1,78-+2,22)$ & $+0,34(-1,72-+1,66)$ & (4) 0,51 \\
\hline (1) Média \pm DP & & & \\
(2) Mediana (mín-máx) & & & \\
(3) Teste t de Student & & & \\
(4) Teste de Mann-Whitney & & &
\end{tabular}

Tabela 3. Resultados dos exames laboratoriais (grupo A).

\begin{tabular}{lccc}
\hline $\begin{array}{l}\text { Dosagem sérica } \\
\mathrm{Ca}^{+2}(\mathrm{mmol} / \mathrm{l})\end{array}$ & Resultados & $\mathbf{n}$ & Valores de Referência \\
Creatinina $(\mathrm{mg} / \mathrm{dl})$ & $1,26 \pm 0,04$ & 59 & $1,15-1,27$ \\
$\mathrm{Pi}(\mathrm{mg} / \mathrm{dl})$ & $0,76 \pm 0,10$ & 60 & $\begin{array}{c}\mathrm{F}=0,6-1,1 \\
\mathrm{M}=0,7-1,2\end{array}$ \\
& $4,7 \pm 0,5$ & 58 & $\begin{array}{c}\text { Crianças: } 4,0-7,0 \\
\text { Adultos: } 2,7-4,5\end{array}$ \\
$\mathrm{FA}(\mathrm{U} / \mathrm{l})$ & $554,7 \pm 142,8$ & 58 & Até 15 anos: menor que 640 \\
$\mathrm{TT}_{4}(\mu \mathrm{g} / \mathrm{dl})$ & $10,4 \pm 2,5$ & 59 & $4,5-12,5$ \\
$\mathrm{FT}_{4}(\mathrm{ng} / \mathrm{dl})$ & $1,69 \pm 0,30$ & 59 & Até 12 anos: $0,65-2,3$ \\
& & & Maiores de 12 anos: $0,8-1,9$ \\
$\mathrm{TSH}(\mu \mathrm{U} / \mathrm{ml})$ & (1) $1,7(0,03-44,0)$ & 59 & $0,4-4,0$
\end{tabular}


Não houve diferença significativa entre a DMO e o CMO dos pacientes que praticavam ou não atividade física regularmente nesta amostra (DMO: $\mathrm{p}=$ 0,11; CMO: $\mathrm{p}=0,32)$.

Também não houve correlação significativa entre DMO e CMO com ingestão diária de cálcio $(\mathrm{r}=$ $0,07$ e $\mathrm{r}=0,04)$, dose média de $l-\mathrm{T}_{4}(\mathrm{r}=0,07$ e $\mathrm{r}=$ $0,10)$, nível médio de TSH $(\mathrm{r}=-0,16 \mathrm{e} \mathrm{r}=-0,23), \mathrm{TT}_{4}$ $(\mathrm{r}=0,01$ e $\mathrm{r}=0,05)$ e $\mathrm{FT}_{4}(\mathrm{r}=-0,14$ e $\mathrm{r}=-0,14)$ durante todo o período de seguimento dos pacientes.

Não houve diferença significativa entre as médias de $\mathrm{DMO}$ e $\mathrm{CMO}$ em relação à etiologia do $\mathrm{HC}$.

Houve correlação positiva tanto da DMO $(\mathrm{r}=$ $0,72)$ como do CMO $(\mathrm{r}=0,72)$ com o IMC, que aumentavam conforme aumentava o IMC.

Para analisar a influência da puberdade na aquisição da massa óssea, separou-se os pacientes de acordo com o sexo. As 23 meninas pré-púberes apresentaram DMO significativamente menor que as 17 meninas púberes $(0,766 \pm 0,060$ vs. $0,845 \pm 0,098$ $\left.\mathrm{g} / \mathrm{cm}^{2} ; \mathrm{p}=0,004\right)$, o mesmo ocorrendo em relação ao CMO $(802,7 \pm 220,7$ vs. $1256,9 \pm 414,9$ g; p < $0,001)$. A mesma análise não foi realizada com os pacientes do sexo masculino, porque o grupo púbere era constituído por apenas dois pacientes.

Não houve correlação entre DMO e CMO e as seguintes variáveis: níveis de $\mathrm{Ca}^{+2}(\mathrm{r}=0,01 \mathrm{e} \mathrm{r}=0,06)$, $\mathrm{Pi}(\mathrm{r}=-0,16$ e $\mathrm{r}=-0,12)$ e FA $(\mathrm{r}=-0,29$ e $\mathrm{r}=-0,24)$.

\section{Comparação entre os grupos A e B}

A tabela 3 mostra o resumo dos dados de comparação do grupo A com o grupo B.

Não se observou diferença estatisticamente significativa entre os sexos, predominando em ambos os grupos crianças do sexo feminino (cerca de 2:1), assim como na prática regular de atividade física entre os grupos (teste qui-quadrado de Pearson, $\mathrm{p}=0,13$ ).
Em ambos os grupos houve predomínio de escolaridade materna e paterna em nível fundamental (cerca de $70 \%)(\mathrm{p}=0,31)$ e a renda familiar per capita também foi semelhante nos dois grupos, predominando a renda inferior a dois salários-mínimos per capita.

Diferença entre os grupos também não foi observada em relação ao estadiamento puberal das mamas (aproximadamente $50 \%$ pré-púberes; $\mathrm{p}=0,60$ ) ou da genitália masculina (cerca de $90 \%$ pré-púberes).

Entretanto, observou-se menor DMO entre os pacientes com $\mathrm{HC}$ (grupo $\mathrm{A}$ ) em relação às crianças e adolescentes saudáveis do grupo B (grupo A: 0,795 \pm 0,075 vs. grupo $\left.\mathrm{B}: 0,832 \pm 0,092 \mathrm{~g} / \mathrm{cm}^{2} ; \mathrm{p}=0,04\right)$, como mostrado na tabela 4 . Não se observou diferença estatisticamente significativa entre os grupos em relação ao $\mathrm{CMO}(959,53 \pm 343,57$ vs. $1080,68 \pm 402,58 \mathrm{~g} ; \mathrm{p}$ $=0,14)$, embora com uma tendência de maior CMO vista nas crianças do grupo B. Também não se encontrou diferença em relação à área corporal total $(1180,95$ $\pm 305,64$ vs. $\left.1268,67 \pm 349,12 \mathrm{~cm}^{2} ; \mathrm{p}=0,23\right)$.

\section{DISCUSSÃo}

Neste estudo, foram avaliados 60 pacientes de 9,9 \pm 1,8 anos de idade com $\mathrm{HC}$ diagnosticado por triagem neonatal e tratado com $l-\mathrm{T}_{4}$ desde $32,8 \pm 19,9$ dias de vida, com dose inicial de $11,7 \pm 1,8 \mu \mathrm{g} / \mathrm{kg} / \mathrm{dia}$ e média de $5,5 \pm 0,8 \mu \mathrm{g} / \mathrm{kg} /$ dia durante o tratamento.

Todos os pacientes tiveram controle laboratorial adequado, o que se refletiu no crescimento, uma vez que os escores- $Z$ da estatura das meninas e dos meninos foram significativamente maiores que os escores-Z da estatura das mães e dos pais.

Uma das limitações do presente estudo foi a impossibilidade da investigação completa do meta-

Tabela 4. Comparação entre os grupos A e B.

\begin{tabular}{lccc}
\hline Parâmetro & $\begin{array}{c}\text { Grupo A } \\
(\mathbf{n}=\mathbf{6 0})\end{array}$ & $\begin{array}{c}\text { Grupo B } \\
(\mathbf{n}=\mathbf{2 8})\end{array}$ & p \\
IC (anos)(1) & $9,9 \pm 1,8$ & $10,4 \pm 2,1$ & $(3) 0,22$ \\
IO (anos)(1) & $9,6 \pm 2,2$ & $10,4 \pm 2,6$ & $(3) 0,18$ \\
Ingestão de cálcio (mg/dia)(1) & $851,2 \pm 466,9$ & $955,4 \pm 482,4$ & $(3) 0,33$ \\
Escore-Z do peso(2) & $+0,21(-1,87-+2,34)$ & $+0,32(-1,83-+1,83)$ & $(4) 0,78$ \\
Escore-Z da estatura(2) & $-0,36(-2,02-+2,79)$ & $-0,32(-1,55-+1,55)$ & $(4) 0,98$ \\
Escore-Z do IMC (2) & $+0,35(-1,78-+2,22)$ & $+0,21(-1,97-+1,94)$ & $(4) 0,98$ \\
Área $\left(\mathrm{cm}^{2}\right)^{(1)}$ & $1180,95 \pm 305,64$ & $1268,67 \pm 349,12$ & $(3) 0,23$ \\
CMO (g)(1) & $959,53 \pm 343,57$ & $1080,68 \pm 402,58$ & $(3) 0,14$ \\
DMO (g/cm²)(1) & $0,795 \pm 0,075$ & $0,832 \pm 0,092$ & $(3) 0,04$ \\
\hline (1) Média \pm DP & & & \\
(2) Mediana (mín-máx) & & & \\
(3) Teste t de Student & & & \\
(4) Teste de Mann-Whitney & & &
\end{tabular}


bolismo do cálcio, como a dosagem dos hormônios calciotrópicos (PTH e vitamina D) e dos marcadores bioquímicos da remodelação óssea.

Hipertireoidismo é descrito como fator de risco para osteoporose, tradicionalmente atribuída aos altos níveis de hormônios tireoideanos (23-25). Recentemente, Abe e cols. (26) propuseram um papel direto do TSH na manutenção da massa óssea ao demonstrar que ratos com deleção do gene do receptor do TSH (TSHR-/-) apresentavam DMO reduzida e que não aumentava após a suplementação com hormônio tireoideano, sugerindo que a perda óssea é independente dos níveis de $\mathrm{T}_{4}$ e $\mathrm{T}_{3}$. Esses autores também sugeriram que o TSH regularia negativamente a remodelação óssea, inibindo a proliferação e a diferenciação, não somente dos osteoclastos, mas também dos osteoblastos por mecanismos distintos (26). Mais tarde, o mesmo grupo (27) demonstrou que o TSH diminui a produção de fator de necrose tumoral $\alpha(\mathrm{TNF} \alpha)$, citocina envolvida na patogênese de várias formas de osteoporose, levando à regulação negativa da osteoclastogênese. Todavia, até o momento ainda não está certo se a perda óssea na tireotoxicose resulta do excesso de hormônio tireoideano, da deficiência de TSH ou da combinação de ambos.

Embora, a dose média de $l-\mathrm{T}_{4}(5,5 \pm 0,8$ $\mu \mathrm{g} / \mathrm{kg} /$ dia) seja maior que a atualmente recomendada para adultos jovens (28) e crianças de 6 a 14 anos (29), e o nível médio de $\mathrm{TT}_{4}(13,1 \pm 1,2 \mu \mathrm{g} / \mathrm{dl})$ esteja ligeiramente acima do limite superior da normalidade, o TSH não estava suprimido no grupo estudado. Poderse-ia especular que o tratamento com doses de $l-\mathrm{T}_{4}$ discretamente maiores pudesse refletir nos valores de DMO dos pacientes em relação ao grupo controle, uma vez que, conforme a metanálise de Uzzan e cols. (30), o excesso de HT aumenta o turnover ósseo e diminui a massa óssea. Como, por questões éticas, não foi dosado $\mathrm{TT}_{4}$ nas crianças do grupo controle, a relação entre DMO e valor de $\mathrm{TT}_{4}$ no grupo A é hipotética, pois não se encontrou correlação entre as médias da dose de $l-\mathrm{T}_{4}$, $\mathrm{TSH}, \mathrm{TT}_{4}$ e $\mathrm{FT}_{4}$ durante todo o seguimento e as médias de $\mathrm{DMO}$ e CMO dos pacientes.

Conforme descrito na literatura (31-33), DMO e CMO aumentaram com a idade e não houve diferença entre os sexos, possivelmente devido ao predomínio de crianças pré-púberes nesta amostra, pois, como já demonstrado, não existe diferença significativa na massa óssea entre os sexos no período prépubertário $(31,34)$.

No presente estudo, as crianças e adolescentes com HC apresentaram DMO significativamente mais baixa que o grupo controle. Este achado não está de acordo com o que foi encontrado por Kooh e cols.
(13) e Léger e cols. (14), que também utilizaram o aparelho Hologic QDR 1000 W. Pitukcheewanont e cols. (15) também não encontraram diferença estatisticamente significativa entre DMO da coluna lombar e do fêmur dos pacientes e dos controles saudáveis de mesmo sexo e idade, utilizando a tomografia computadorizada quantitativa.

Entretanto, esses autores avaliaram apenas a DMO da coluna lombar e do fềmur num número menor de crianças pré-púberes. Assim como esses, outros estudos em crianças e adolescentes com HC não utilizaram densitometria óssea de corpo total pela DXA, que já foi validada e considerada, até o momento, como a melhor forma de avaliar o estado mineral ósseo durante o crescimento e a maturação sexual $(35,36)$. Por isso, a comparação dos resultados obtidos nos pacientes desta amostra com os da literatura fica prejudicada.

No entanto, Salerno e cols. (16) encontraram redução da DMO da coluna lombar em 13,5\% dos adolescentes (média de 17,8 anos de idade) estudados pela DXA e 38\% deles ao realizar ultra-sonografia quantitativa (QUS) da segunda à quinta falange proximal da mão não-dominante. Os resultados obtidos nesta amostra não são comparáveis aos do trabalho de Salerno e cols. (16), dadas as diferenças de métodos utilizados e a faixa etária dos pacientes.

Assim como no presente estudo, Kooh e cols. (13), Léger e cols. (14), Salerno e cols. (16) e Pitukchhewanont e cols. (15) também não encontraram diferença significativa de $\mathrm{DMO}$ em relação à etiologia do $\mathrm{HC}$, e doses elevadas de $l$ - $\mathrm{T}_{4}$ não tiveram influência sobre a massa óssea.

A DMO e o CMO dos pacientes incluídos neste estudo aumentaram conforme o aumento do IMC e, de acordo com Frost (37) e Kiss (38), adultos obesos tendem a ter maior DMO, apesar de que isto só ocorre quando o indivíduo obeso não é sedentário. Como os escores- $Z$ de peso e IMC foram semelhantes entre os pacientes e os controles, obesidade não pode ser considerada como determinante de maior DMO entre os indivíduos do grupo B.

A importância dos esteróides sexuais, principalmente dos estrogênios, na aquisição da massa óssea já foi descrita na literatura (39-41) e, de acordo com este achado, neste estudo as meninas púberes apresentaram DMO e CMO mais altos que as meninas pré-púberes. A influência da puberdade no sexo masculino não foi analisada, devido à presença de apenas dois meninos púberes na amostra. Entretanto, como o número de crianças púberes e pré-púberes foi semelhante nos grupos A e B, a puberdade não foi o fator relacionado à maior $\mathrm{DMO}$ entre os controles. 
O sedentarismo está entre os fatores de risco para a aquisição de um menor pico de massa óssea (24). Apesar de $76,7 \%$ dos pacientes com HC (grupo A) não praticarem atividade física regularmente, não houve diferença entre os grupos $\mathrm{A}$ e $\mathrm{B}$, também não sendo esta a variável determinante da menor média de DMO no grupo A. Da mesma forma, o nível de escolaridade dos pais e a renda familiar per capita foram semelhante entre os grupos, não sendo o fator sócio-econômico o responsável pela redução de $\mathrm{DMO}$ observada nesses pacientes.

Ingestão de cálcio já foi correlacionada à $\mathrm{DMO}$ em crianças e adolescentes. Estudos têm demonstrado que dieta pobre em cálcio pode afetar a aquisição de massa óssea, enquanto que aumento da DMO foi observado ao se utilizar suplementos de cálcio $(42,43)$. Assim como no estudo de Salerno e cols. (16), a maioria $(65 \%)$ dos pacientes desta amostra tinha ingestão diária de cálcio inferior à quantidade atualmente recomendada, mas não se encontrou DMO mais baixa nas crianças que ingeriam menos cálcio, achado semelhante ao de Salerno e cols. (16) e diferente do de Léger e cols. (14). No entanto, no presente estudo, a ingestão foi semelhante entre os grupos, também não sendo a ingestão inadequada de cálcio a determinante da menor DMO do grupo A em relação ao grupo B.

\section{CONCLUSÕES}

Este estudo transversal mostrou que a DMO de corpo total foi significativamente menor no grupo com HC do que no grupo controle, resultado este que poderia ser explicado pelo método diagnóstico empregado no estudo ou, possivelmente, relacionado ao tratamento. Após uma extensa busca na literatura, identificamos que o presente estudo é o pioneiro em avaliar a DMO de corpo total em pacientes com HC, que é o método considerado atualmente como o mais adequado para avaliar a massa óssea em crianças e adolescentes.

Os dados obtidos neste estudo indicam a necessidade de acompanhar os pacientes até a idade adulta, bem como ampliar a investigação da homeostase do cálcio em crianças com $\mathrm{HC}$ em tratamento, visando ao esclarecimento da patogênese da redução da massa óssea.

\section{AGRADECIMENTOS}

Os autores agradecem à técnica Janaína Bonoldi pelo imprescindível trabalho de realização das densitometrias ósseas, ao Serviço de Análises Clínicas do HCUFPR e a todo o Corpo Clínico e Técnico da UEP.

\section{REFERÊNCIAS}

1. Kopp P. Perspective: genetic defects in the etiology of congenital hypothyroidism. Endocrinology 2002;143:2019-24.

2. Brown RS, Demmer LA. Editorial: the etiology of thyroid dysgenesis - still an enigma after all these years. J Clin Endocrinol Metab 2002;87:4069-71.

3. Harvey CB, O'Shea PJ, Scott AJ, Robson H, Siebler T, Shalet $\mathrm{SM}$, et al. Molecular mechanisms of thyroid hormone effects on bone growth and function. Mol Genet Metab 2002;75:17-30.

4. Basset JHD, Williams GR. The molecular actions of thyroid hormone in bone. Trends Endocrinol Metab 2003;14:35664.

5. Rizzoli R, Poser J, Bürgi U. Nuclear thyroid hormone receptors in cultured bone cells. Metabolism 1986;35:71-4.

6. Robson H, Siebler T, Stevens DA, Shalet SM, Williams GR. Thyroid hormone acts directly on growth plate chondrocytes to promote hypertrophic differentiation and inhibit clonal expansion and cell proliferation. Endocrinology 2000;141:3887-97.

7. Lakatos P, Foldes J, Horvath C, Kiss L, Tatrai A, Takacs I, et al. Serum interleukin- 6 and bone metabolism in patients with thyroid function disorders. J Clin Endocrinol Metab 1997:82:78-81.

8. Carey $\mathrm{DE}$, Jones $\mathrm{KL}$, Parthemore JG, Deftos LJ. Calcitonin secretion in congenital nongoitrous cretinism. J Clin Invest 1980;65:892-5.

9. Zamboni G, Avanzini S, Giavarina D, Tato L. Monomeric calcitonin secretion in infants with congenital hypothyroidism. Acta Paediatr Scand 1989;78:885-8.

10. Demeester-Mirkine N, Bergmann P, Body J-J, Corvilain J. Calcitonin and bone mass status in congenital hypothyroidism. Calcif Tissue Int 1990;46:222-6.

11. Body JJ, Chanoine JP, Dumon JC, Delange F. Circulating calcitonin levels in healthy children and subjects with congenital hypothyroidism from birth to adolescence. J Clin Endocrinol Metab 1993;77:565-7.

12. Daripa M, Paula FJD, Rufino ACB, Foss MC. Impact of congenital calcitonin deficiency due to dysgenetic hypothyroidism on bone mineral density. Braz $\mathbf{J}$ Med Biol Res 2004;37:61-8.

13. Kooh SW, Brnjac L, Ehrlich RM, Qureshi R, Krishnan S. Bone mass in children with congenital hypothyroidism treated with thyroxine since birth. J Pediatr Endocrinol Metab 1996;9:59-62.

14. Léger J, Ruiz JC, Guibourdenche J, Kindermans C, Garabedian M, Czernichow P. Bone mineral density and metabolism in children with congenital hypothyroidism after prolonged Lthyroxine therapy. Acta Paediatr 1997;86:704-10.

15. Pitukcheewanont $P$, Safani D, Gilsanz V, Klein M, Chongpison $Y$, Costin G. Quantitative computed tomography measurements of bone mineral density in prepubertal children with congenital hypothyroidism treated with L-thyroxine. J Pediatr Endocrinol Metab 2004;17:889-93.

16. Salerno $S$, Lettiero $T$, Esposito-del Puente A, Esposito $V$, Capalbo D, Carpinelli A, et al. Effect of long-term L-thyroxine treatment on bone mineral density in young adults with congenital hypothyroidism. Eur J Endocrinol 2004;151:689-94.

17. National Center for Health Statistics (NCHS). Clinical growth charts 2000. Disponível em: <http://www.cdc.gov/ nchs/about/major/nhanes/growthcharts/clinical charts.htm $>$.

18. Tanner JM. Growth at adolescence. $2^{\text {nd }}$ ed. Oxford: Blackwell, 1962.

19. Prader A. Testicular size: assessment and clinical importance. Triangle 1966;7:240-3.

20. Thériault D. BMD Patient intake questionnaire 2004. Disponível em: <http://www.iscd.org/visitors/resources/ BMDQuestionaire.doc>.

21. Institute of Medicine, The Food and Nutrition Board. Dietary references intakes for calcium, phosphorus, magnesium, vitamin D, and fluoride. Washington, DC: National Academies Press, 1997. 
22. Greulich WW, Pyle SI. Radiographic atlas of skeletal development of the hand and wrist. $2^{\text {nd }}$ ed. Stanford: Stanford University Press, 1959.

23. Baran DT. Editorial: detrimental skeletal effects of thyrotropin suppressive doses of thyroxine: fact or fantasy? J Clin Endocrinol Metab 1994;78:816-7.

24. National Institutes of Health. Osteoporosis prevention, diagnosis and therapy: Consensus Conference. JAMA 2001;285:785-95.

25. Lakatos P. Thyroid hormones: beneficial or deleterious for bone? Calcif Tissue Int 2003;73:205-9.

26. Abe E, Marians RC, Yu W, Xue-Bin W, Ando T, Li Y, et al. TSH is a negative regulator of skeletal remodeling. Cell 2003;115:151-62.

27. Hase $H$, Ando T, Eldeiry L, Brebene A, Yuanzhen P, Liu L, et al. TNF $\alpha$ mediates the skeletal effects of thyroid-stimulating hormone. Proc Natl Acad Sci U S A 2006;103:12849-54 (Disponível online em: <http://www.pnas.org/cgi/doi/10.1073/ pnas.0600427103>).

28. Singer PA, Cooper DS, Levy EG, Ladenson PW, Braverman $\mathrm{LE}$, Daniels $\mathrm{G}$, et al. Treatment guidelines for patients with hyperthyroidism and hypothyroidism. Standards of Care Committee, American Thyroid Association. JAMA 1995;273:808-12.

29. BRASIL. Ministério da Saúde. Portaria no 848, 2002. Disponível em: <http://dtr2001.saude.gov.br/sas/PORTARIAS/PORT2002/PT-848.htm>.

30. Uzzan B, Campos J, Cucherat M, Nony P, Boissel JP, Perret GY. Effects on bone mass of long term treatment with thyroid hormones: a meta-analysis. J Clin Endocrinol Metab 1996;81:4278-89.

31. Glastre G, Braillon P, David L, Cochat P, Meunier PJ, Delmas PD. Measurement of bone mineral content of lumbar spine by dual energy X-ray absorptiometry in normal children: correlations with growth parameters. J Clin Endocrinol Metab 1990:70:1330-3.

32. Carrascosa $A$, Gussinyé $M$, Yeste $D$, del Rio L, Audí L, Enrubia $M$, et al. Skeletal mineralization during infancy, childhood and adolescence in the normal population and in populations with nutricional and hormonal disorders. Dual X-ray absorptiometry (DEXA) evaluation. In: Schönau E. Paediatric Osteology. $1^{\text {st }}$ ed. Amsterdam: Elsevier, 1996. pp. 93-102.

33. Fonseca ASM, Szejnfeld VL, Terreri MT, Goldenberg J, Ferraz $M B$, Hilário MOE. Bone mineral density of the lumbar spine of Brazilian children and adolescents aged 6 to 14 years. Braz J Med Biol Res 2001;34:347-52.
34. Bonjour JP, Theintz G, Buchs B, Slosman D, Rizzoli R. Critical years and stage of puberty for spinal and femoral bone mass accumulation during adolescence. J Clin Endocrinol Metab 1991;73:555-63.

35. Ellis KJ, Shypailo RJ, Pratt JA, Pond WG. Accuracy of dualenergy $\mathrm{x}$-ray absorptiometry for body-composition measurements in children. Am J Clin Nutr 1994;60:660-5.

36. Molgaard C, Thomsen BL, Prentice A, Cole TJ, Michaelsen $\mathrm{KF}$. Whole body bone mineral content in healthy children and adolescents. Arch Dis Child 1997;76:9-15.

37. Frost HM. Obesity, and bone strength and "mass": a tutorial based on insights from a new paradigm. Bone 1997;21:211-

38. Kiss MHB. Osteoporose. In: Setian N. Endocrinologia Pediátrica: aspectos físicos e metabólicos do recémnascido ao adolescente. $2^{\mathrm{a}}$ ed. São Paulo: Sarvier, 2002. pp. 354-62.

39. Boot AM, Ridder MAJ, Pols HAP, Krenning EP, Keizer-Schrama SPFM. Bone mineral density in children and adolescents: relation to puberty, calcium intake, and physical activity. $\mathbf{J}$ Clin Endocrinol Metab 1997;82:57-62.

40. Slemenda CW, Reister TK, Hui SL, Miller JZ, Christian JC, Johnston Jr. CC. Influences on skeletal mineralization in children and adolescents: evidence for varying effects of sexual maturation and physical activity. J Pediatr 1994;125:201-7.

41. Soyka LA, Fairfield WP, Klibanski A. Hormonal determinants and disorders of peak bone mass in children. J Clin Endocrinol Metab 2000;85:3951-62.

42. Chan GM. Dietary calcium and bone mineral status of children and adolescents. Am J Dis Child 1991;145:631-4.

43. Bonjour JP, Carrie AL, Ferrari S, Clavien H, Slosman D, Theintz G, et al. Calcium-enriched foods and bone mass growth in prepubertal girls: a randomized, double-blind, placebo-controlled trial. J Clin Invest 1997;9:1287-94.

Endereço para correspondência:

Adriane de André Cardoso Demartini

Av. Romeu Strazzi 1744, ap. 11 B

15085-520 São José do Rio Preto, SP

E-mail: dra.adriane@yahoo.com.br 